\title{
Exact Imaging by an Elliptic Lens
}

\author{
Oguzhan Akgol, Danilo Erricolo, Senior Member, IEEE, Piergiorgio L. E. Uslenghi, Life Fellow, IEEE
}

\begin{abstract}
The radiation from an isotropic line source located at one of the focal lines of an elliptic cylindrical lens filled with a homogeneous and lossless DNG metamaterial is determined exactly, in the frequency domain. This exact solution is compared to a previously derived optical solution.
\end{abstract}

Index Terms-Electromagnetic radiation, lenses, Mathieu functions, metamaterial.

\section{INTRODUCTION}

D OUBLE-negative (DNG) metamaterials whose permittivity and permeability have real negative values at some frequencies have attracted considerable attention in recent years. In particular, the imaging of an isotropic line source located at one of the focal lines of an elliptic cylindrical lens made of DNG metamaterial has been studied in the optical limit [1]. It has been shown that the line source is imaged onto a non-isotropic virtual line source located at the other focal line of the lens. In this work, an exact electromagnetic solution of the same problem is obtained as an infinite series of ellipticcylinder wave functions, each term of the series consisting of products of radial and angular Mathieu functions. The modal expansion coefficients are determined analytically by imposing the boundary conditions. The exact radiated far field is obtained, and is evaluated numerically by computing the series of eigenfunctions. The numerical results are compared with the optical limit obtained in [1]. Some preliminary results were previously presented at a conference [2].

The double-positive (DPS) material filling the infinite space outside the lens is characterized by a real positive electric permittivity $\epsilon$ and a real positive magnetic permeability $\mu$, while the corresponding parameters inside the DNG lens are a real negative permittivity $-\epsilon$ and a real negative permeability $-\mu$. As a consequence of causality, the refractive index outside the lens is real positive, and is the opposite of the real negative refractive index inside the lens, while the intrinsic impedance has the same real positive value $Z=\sqrt{(\mu / \epsilon)}$ everywhere [3].

The time-dependence factor $\exp (+j \omega t)$ is omitted throughout. The wave number is $k=\omega \sqrt{(\epsilon \mu)}$ in the DPS medium and $-k$ in the DNG material.

\section{Geometry of The PROBlem}

A cross-sectional view of the structure in a plane $z=$ constant is shown in Fig. 1.

The authors are with the Department of Electrical and Computer Engineering, University of Illinois at Chicago, 851 South Morgan St., Chicago, Illinois 60607, USA. E-mail: oguzhanakgoluni@yahoo.com, erricolo@ece.uic.edu, uslenghi@uic.edu.

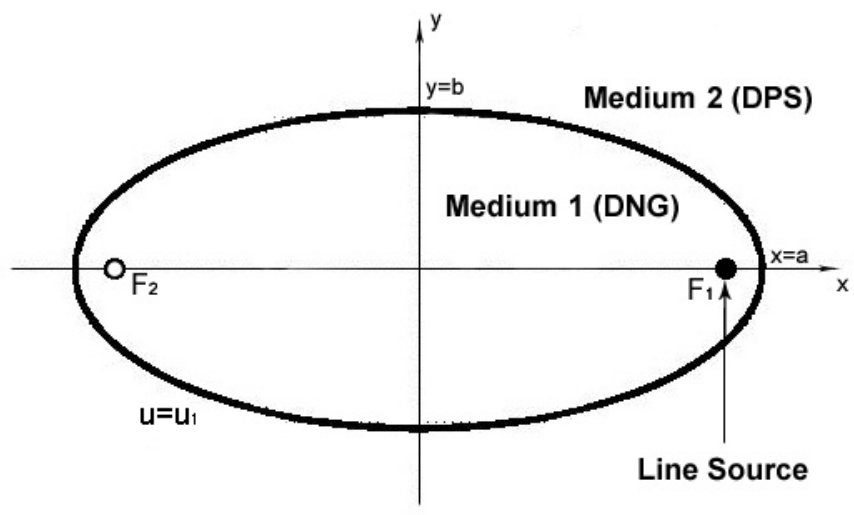

Fig. 1. Cross section of the geometry of the problem.

The rectangular coordinates $(x, y, z)$ are related to the elliptic cylinder coordinates

$$
\begin{aligned}
x & =\frac{d}{2} \cosh u \cos v, \\
y & =\frac{d}{2} \sinh u \sin v, \\
z & =z
\end{aligned}
$$

where $d$ is the interfocal distance, $0 \leq u<\infty, 0 \leq v \leq 2 \pi$, and $-\infty<z<\infty$. Curves $u=$ constant are ellipses and curves $v=$ constant are hyperbolas. We define the dimensionless real positive parameter

$$
c=\frac{k d}{2}=\frac{\pi d}{\lambda}
$$

where $\lambda$ is the wavelength.

\section{LINE SOURCE INCIDENCE}

\section{A. E-polarization}

Consider an electric line source parallel to the z-axis and located at $\left(x_{0}, y_{0}\right) \equiv\left(u_{0}, v_{0}\right)$ inside the DNG lens, whose primary electric field is

$$
\boldsymbol{E}^{i}=\hat{z} E_{z}^{i}=\hat{z} H_{0}^{(2)}(-k R)
$$

where

$$
R=\sqrt{\left(x-x_{0}\right)^{2}+\left(y-y_{0}\right)^{2}}
$$

is the distance between the line source and the observation point $(x, y) \equiv(u, v)$ and $H_{0}^{(2)}$ is the Hankel function of the second kind. The incident field may be expanded in a series 
of elliptic-cylinder wave functions [4]-[5]:

$$
\begin{aligned}
& E_{1 z}^{i}=H_{0}^{(2)}(-k R)=4 \sum_{m=0}^{\infty} \\
& {\left[\frac{1}{N_{m}^{(e)}} \operatorname{Re}_{m}^{(1)}\left(-c, u_{<}\right) \operatorname{Re}_{m}^{(4)}\left(-c, u_{>}\right) \operatorname{Se}_{m}\left(-c, v_{0}\right) \mathrm{Se}_{m}(-c, v)+\right.} \\
& \left.\frac{1}{N_{m}^{(o)}} \operatorname{Ro}_{m}^{(1)}\left(-c, u_{<}\right) \operatorname{Ro}_{m}^{(4)}\left(-c, u_{>}\right) \mathrm{So}_{m}\left(-c, v_{0}\right) \mathrm{So}_{m}(-c, v)\right]
\end{aligned}
$$

where $u_{<}\left(u_{>}\right)$is the smaller (larger) between $u$ and $u_{0}$. When the electric line source is located inside the lens at the focal line $\left(x_{0}=d / 2, y_{0}=0\right)$ the incident electric field only contains even functions and it becomes

$$
E_{1 z}^{i}=4 \sum_{m=0}^{\infty} \frac{1}{N_{m}^{(e)}} \operatorname{Re}_{m}^{(1)}(-c, 0) \operatorname{Re}_{m}^{(4)}(-c, u) \operatorname{Se}_{m}(c, v)
$$

because of properties of Mathieu functions [5] and the fact that the angular Mathieu functions $\mathrm{Se}_{m}$ and $\mathrm{So}_{m}$ are even functions of the parameter $c$ has been taken into account. It should be noted that the Mathieu radial functions are not even functions of $c$ [6]. The scattered field inside the lens is

$$
E_{1 z}^{s}=4 \sum_{m=0}^{\infty} \frac{a_{m}}{N_{m}^{(e)}} \operatorname{Re}_{m}^{(1)}(-c, u) \operatorname{Se}_{m}(c, v) .
$$

Outside the lens, the scattered field is

$$
E_{2 z}^{s}=4 \sum_{m=0}^{\infty} \frac{b_{m}}{N_{m}^{(e)}} \operatorname{Re}_{m}^{(4)}(c, u) \operatorname{Se}_{m}(c, v),\left(u \geq u_{1}\right)
$$

and it satisfies the radiation condition because it contains the radial functions of the fourth kind, $\operatorname{Re}_{m}^{(4)}$. The modal expansion coefficients $a_{m}^{(e)}$ and $b_{m}^{(e)}$, are obtained by imposing the boundary conditions on the continuity of the total tangential components of $\mathbf{E}$ and $\mathbf{H}$ across the interface at $u=u_{1}$, yielding

$$
\begin{aligned}
a_{m}= & \frac{-\operatorname{Re}_{m}^{(1)}(-c, 0)}{\Delta}\left[\operatorname{Re}_{m}^{(4)^{\prime}}\left(c, u_{1}\right) \operatorname{Re}_{m}^{(4)}\left(-c, u_{1}\right)+\right. \\
b_{m}= & j \frac{\left.\operatorname{Re}_{m}^{(4)}\left(c, u_{1}\right) \operatorname{Re}_{m}^{(4)^{\prime}}\left(-c, u_{1}\right)\right]}{\Delta},
\end{aligned}
$$

where

$$
\Delta=\operatorname{Re}_{m}^{(1)}\left(-c, u_{1}\right) \operatorname{Re}_{m}^{(4)^{\prime}}\left(c, u_{1}\right)+\operatorname{Re}_{m}^{(1)^{\prime}}\left(-c, u_{1}\right) \operatorname{Re}_{m}^{(4)}\left(c, u_{1}\right)
$$

and the prime means the derivative with respect to the argument $u$.

\section{B. H-polarization}

The analysis is similar to that for E-polarization. For a magnetic line source located at $F_{1}$ in Fig.1, the incident magnetic field is

$$
\mathbf{H}^{\mathbf{i}}=\hat{\mathbf{z}} H_{z}^{i}
$$

with

$$
\begin{aligned}
& H_{1 z}^{i}= \\
& 4 \sum_{m=0}^{\infty} \frac{1}{N_{m}^{(e)}} \operatorname{Re}_{m}^{(1)}(-c, 0) \operatorname{Re}_{m}^{(4)}(-c, u) \operatorname{Se}_{m}(c, v) .
\end{aligned}
$$

The scattered magnetic field inside the lens is

$$
H_{1 z}^{s}=4 \sum_{m=0}^{\infty} \frac{c_{m}}{N_{m}^{(e)}} \operatorname{Re}_{m}^{(1)}(-c, u) \operatorname{Se}_{m}(c, v),
$$

and the total magnetic fields outside the lens is

$$
H_{2 z}^{s}=4 \sum_{m=0}^{\infty} \frac{d_{m}}{N_{m}^{(e)}} \operatorname{Re}_{m}^{(4)}(c, u) \operatorname{Se}_{m}(c, v)
$$

The modal coefficients are obtained from the application of the boundary conditions and, because of duality, one finds $c_{m}=a_{m}$ and $d_{m}=b_{m}$.

\section{Far Field Expressions}

For E polarization, the scattered field is given by equation (10). When the Mathieu radial function $\operatorname{Re}_{m}^{(4)}(c, u)$ is replaced by its asymptotic expression $j^{m} \exp (-j k \rho+j \pi / 4) \sqrt{k \rho}$, one finds

$$
E_{2 z}^{s} \approx 4 \frac{\exp (-j k \rho+j \pi / 4)}{\sqrt{k \rho}} \sum_{m=0}^{\infty} b_{m} \frac{j^{m}}{N_{m}^{(e)}} S e_{m}(c, v) .
$$

In order to obtain results that are not dependent upon the distance $\rho$ from the origin, we divide the previous expression by $\exp (-j k \rho+\pi / 4) / \sqrt{k \rho}$ so that we obtain the far field radiation pattern

$$
P(v)=4 \sum_{m=0}^{\infty} b_{m} \frac{j^{m}}{N_{m}^{(e)}} S e_{m}(c, v) .
$$

The same far field pattern holds for $\mathrm{H}$ polarization.

\section{NUMERICAL RESULTS}

We compare the far-field radiation pattern of eq. (19) with the geometrical optics solution presented in [1]. For this purpose, we consider three ellipses with $u_{1}=0.5, u_{1}=0.6$, and $u_{1}=0.8$. Each ellipse is represented normalized to its major axis $2 a$ in Fig. 2, which emphasizes also the variation in eccentricity

$$
\chi=\frac{d}{2 a}=\frac{1}{\cosh u_{1}}
$$

by showing a comparison with a circle. The ellipses have major axes with different lengths so that we compare numerical results in terms of the ratio between the wavelength and the major axis, i.e.

$$
r=\frac{2 a}{\lambda}=\frac{d \cosh u_{1}}{\lambda} .
$$

Hence, the eccentricity controls the different behavior of the solutions corresponding to the same value of the ratio $r$. The 


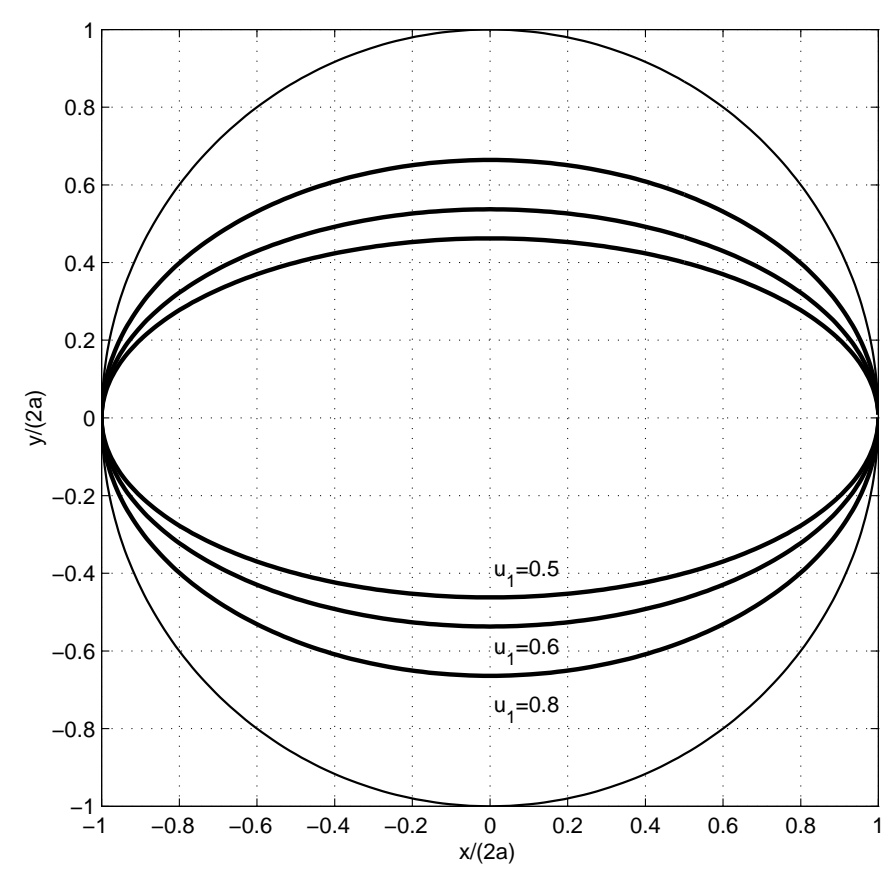

Fig. 2. Normalized cross sections of the ellipses considered in the numerical examples and comparison with a circle.

solution presented in [1] is formulated in terms of the power emitted per unit angle, computed as

$$
p(v)=\frac{1-\chi^{2}}{1+\chi^{2}-2 \chi \cos v},
$$

which corresponds to $r=\infty$. Therefore the previous formula is compared with the magnitude square of the far-field radiation pattern (19), i.e.

$$
|P(v)|^{2}=16\left|\sum_{m=0}^{\infty} b_{m} \frac{j^{m}}{N_{m}^{(e)}} S e_{m}(c, v)\right|^{2} .
$$

Fig. 3 shows the normalized polar plot of $|P(v)|^{2}$ and the geometrical optics solution $p(v)$ for an ellipse with $u_{1}=0.5$ and eccentricity $\chi=0.8868$. A second set of results is shown in Fig. 4 for an ellipse with $u_{1}=0.6$ and eccentricity $\chi=$ 0.844. A third set of results is shown in Fig. 5 for an ellipse with $u_{1}=0.8$ and eccentricity $\chi=0.748$. In all cases, when the ellipse is electrically small, $r=0.1$, the far-field result is significantly different from the geometrical optics solution and it is closer to an omnidirectional pattern. However, when the ellipse becomes electrically larger, a better agreement with the geometrical optics solution (corresponding to $(r=\infty)$ is observed.

Finally, all the numerical results correspond to the summation of up to the first 40 terms of the series (23) and the evaluation of Mathieu function was accomplished using an extension of the Fortran code described in [7] combined with the acceleration technique [8]. The series (23) converges slowly and one needs to stop the summation before numerical instabilities occur.

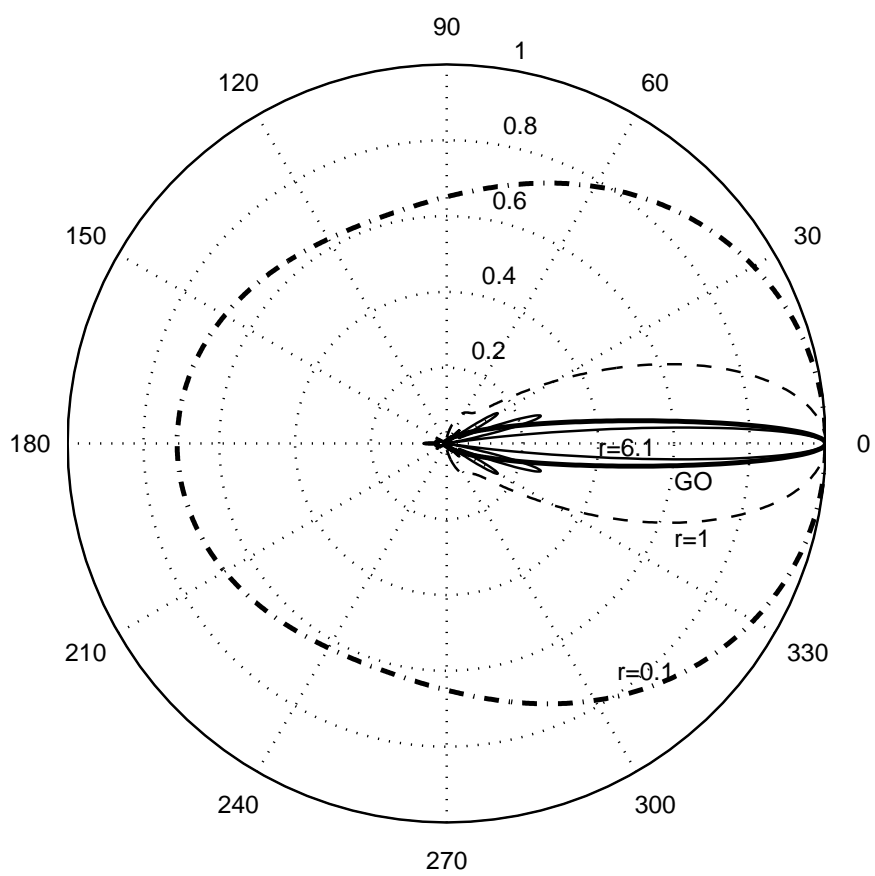

Fig. 3. Comparison between the far-field pattern $|P(v)|^{2}$ and the geometrical optics solution $p(v)$ of eq. (22) computed for an ellipse with $u_{1}=0.5$. The values are normalized to their maxima and plotted on a linear scale. The results represent: far-field pattern for $r=0.1$ or $c=0.235$ (dash-dot line); far-field pattern for $r=1$ or $c=2.348$ (dashed line); far-field pattern for $r=6.1$ or $c=16.995$ (thin solid line); and, geometrical optics solution (thick solid line, $r=\infty$ ).

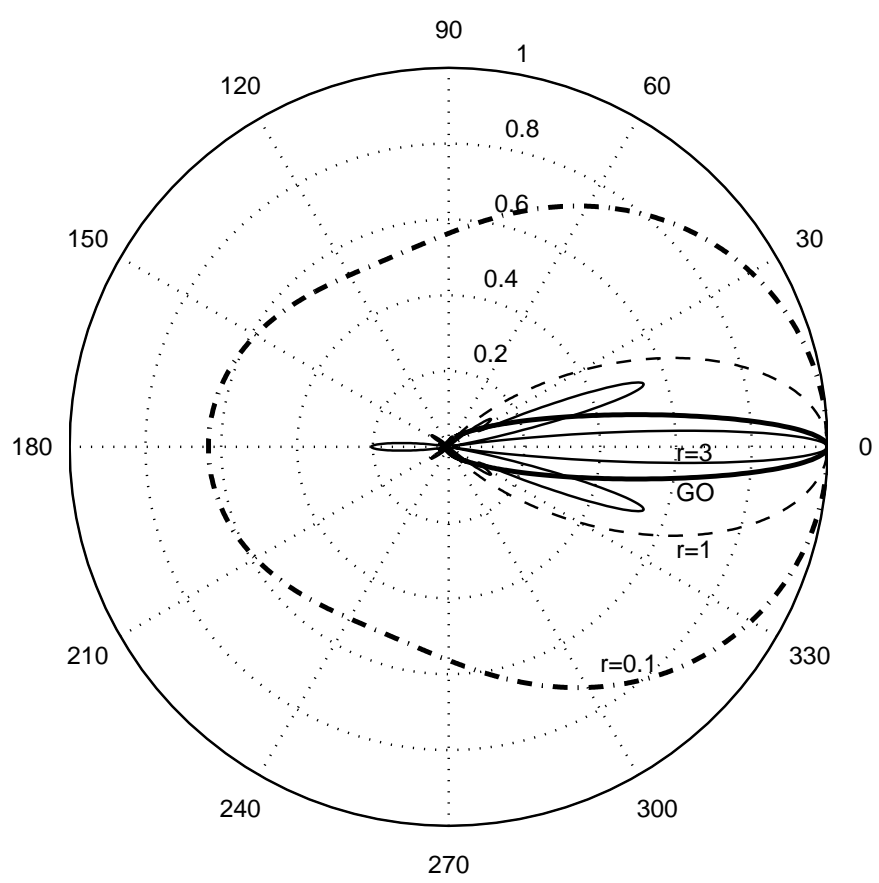

Fig. 4. Comparison between the far-field pattern $|P(v)|^{2}$ and the geometrical optics solution $p(v)$ of eq. (22) computed for an ellipse with $u_{1}=0.6$. The values are normalized to their maxima and plotted on a linear scale. The results represent: far-field pattern for $r=0.1$ or $c=0.235$ (dash-dot line); far-field pattern for $r=1$ or $c=2.348$ (dashed line); far-field pattern for $r=5.1$ or $c=13.5155$ (thin solid line); and, geometrical optics solution (thick solid line, $r=\infty$ ). 


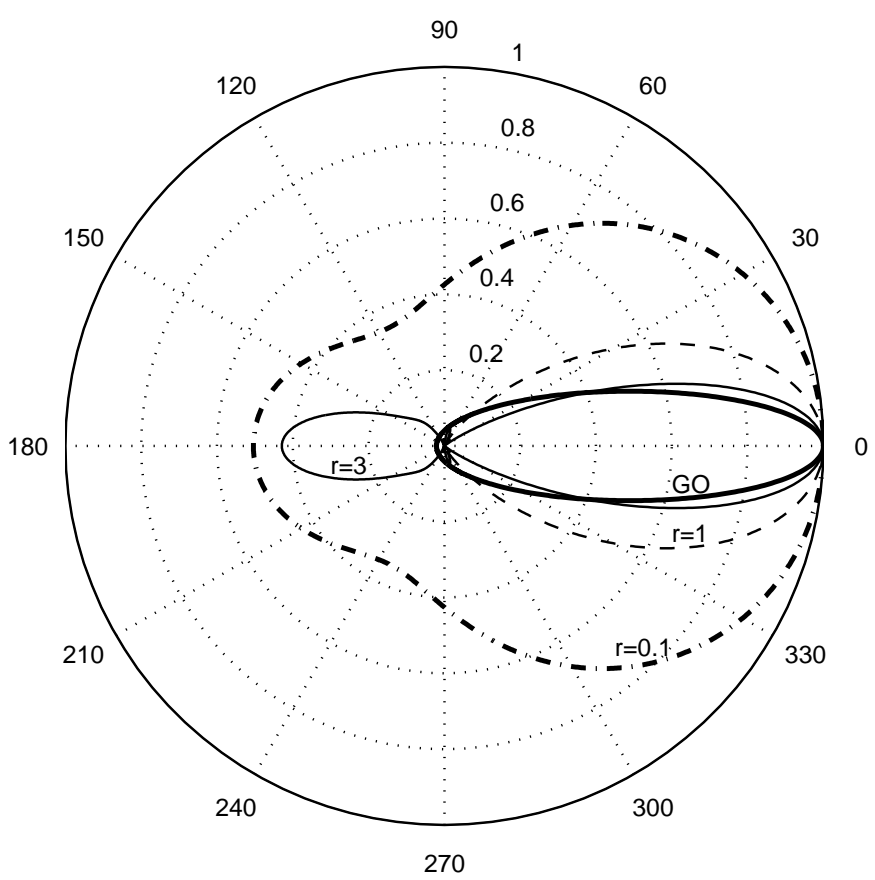

Fig. 5. Comparison between the far-field pattern $|P(v)|^{2}$ given by eq. (23) and the geometrical optics solution $p(v)$ of eq. (22) computed for an ellipse with $u_{1}=0.8$. The values are normalized to their maxima and plotted on a linear scale. The results represent: far-field pattern for $r=0.1$ or $c=0.235$ (dash-dot line); far-field pattern for $r=1$ or $c=2.348$ (dashed line); farfield pattern for $r=3$ or $c=7.047$ (thin solid line); and, geometrical optics solution (thick solid line, $r=\infty$ ).

\section{CONCLUSIONS}

The exact electromagnetic solution for the two-dimensional problem of a DNG lens fed by a line source located at one focal point was compared with the geometrical optics solution of the same problem. The numerical comparison show that the agreement with the geometrical optics solution improves for electrically large ellipses and, for the same value of the ratio $r$, the agreement is better when the ellipse has a smaller eccentricity.

This solution extends also the list of exact canonical solutions of boundary value electromagnetic scattering problems involving metamaterials.

\section{REFERENCES}

[1] P.L.E. Uslenghi, "Optical behavior of elliptical lenses made of DNG metamaterial," IEEE Antennas and Wireless Propag. Lett., vol.9, pp. 566-567, 2010,

[2] O. Akgol, D. Erricolo and P.L.E. Uslenghi, "Electromagnetic behavior of an elliptical lens made of DNG metamaterial", Proc. XXIX General Assembly of the International Union of Radio Science (URSI), Chicago, Illinois, August 11-15,2008.

[3] Ziolkowski, R. W., and E. Heyman, "Wave propagation in media having negative permittivity and permeability," Phys. Rev. E, 64(5), 056,625, doi:10.1103/PhysRevE.64.056625, 2001.

[4] J.J. Bowman, T.B.A. Senior, and P.L.E. Uslenghi, Electromagnetic and Acoustic Scattering by Simple Shapes, Hemisphere Publishing Corporation, New York, 1987.

[5] P.L.E. Uslenghi, "Exact penetration, radiation and scattering for a slotted semielliptical channel filled with isorefractive material," IEEE Trans. Antennas Propag., vol.52, no. 6 pp. 1473-1480, June 2004,
[6] O. Akgol, D. Erricolo and P.L.E. Uslenghi, "Electromagnetic Radiation and Scattering for a Gap in a Corner Backed by a Cavity Filled with DNG Metamaterial," Radio Science, 2011, accepted,

[7] D. Erricolo, "Algorithm 861: Fortran 90 subroutines for computing the expansion coefficients of Mathieu functions using Blanch's algorithm," ACM Transactions on Mathematical Software, vol. 32, no. 4, pp. 622634, Dec. 2006.

[8] D. Erricolo, "Acceleration of the convergence of series containing Mathieu functions using Shanks transformation," IEEE Antennas Wireless Propag. Lett., vol. 2, pp. 58-61, 2003. 